\title{
EL APRENDIZAJE BASADO EN PROYECTOS: UNA CAPACITACIÓN DOCENTE VINCULANTE
}

\author{
Project-Based Learning: A Binding Teacher Training
}

\author{
Ernesto Cyrulies ${ }^{1}$ \\ ORCID: 0000-0002-2080-5864 \\ Mariana Schamne ${ }^{2}$ \\ ORCID: 0000-0003-0685-4974 \\ ${ }^{1}$ Universidad Nacional de General Sarmiento, Argentina. \\ ${ }^{12}$ Centro de Capacitación, Información e Investigación Educativa Malvinas Argentinas, Argentina. \\ Correos: ecyrulie@campus.ungs.edu.ar; schamnemariana@gmail.com
}

Recibido: 27/09/2020

Aceptado: 24/12/2020

Resumen: En este trabajo se describe una experiencia que incluye el diseño, los procedimientos y los resultados de un curso de capacitación docente sobre aprendizaje basado en proyectos (ABP), impartido por un centro de capacitación oficial de la provincia de Buenos Aires, Argentina. La oferta estuvo dirigida a docentes de diversos niveles y áreas de desempeño, que conformaron grupos de aprendizaje heterogéneos para promover un trabajo con transversalidad curricular. Se desarrolla un ejemplo de proyecto modélico utilizado en el curso y se analizan las producciones docentes elaboradas para acreditarlo y los resultados de la aplicación de una encuesta. El registro de la experiencia tuvo como objeto documentar información de interés, particularmente para futuras capacitaciones e investigaciones educativas. Los resultados muestran una gran aceptación del ABP, pero se identifican dificultades en su implementación, particularmente por las condiciones institucionales de trabajo.

Palabras clave: capacitación docente; aprendizaje basado en proyectos; transversalidad curricular; aprendizaje cooperativo.

\begin{abstract}
This paper describes an experience that includes the design, procedures and results of a teacher training course on project-based learning (PBL), offered by an official training center in the province of Buenos Aires, Argentina. The course was aimed at teachers from different levels and areas of performance, who formed heterogeneous learning groups to promote work with curricular transversality. This article develops an example of a model project used in the course and analyzes the teachers' productions elaborated to accredit the course and the results of the application of a survey. The purpose of recording the experience was to document information of interest, particularly for future training and educational research. The results show a great acceptance of PBL, but difficulties are identified in its implementation, particularly due to the institutional working conditions.
\end{abstract}

Keywords: teacher training; project-based learning; curricular transversality; cooperative learning. 


\section{Introducción}

La Dirección de Formación Docente Permanente del Ministerio de Educación de la provincia de Buenos Aires ofrece cursos de capacitación docente para distintas áreas curriculares y para diferentes niveles educativos (inicial, primario y secundario) a través de los Centros de Capacitación Información e Investigación Educativa (CIIE). Estas ofertas de formación permanente ponen la mirada en los aspectos didácticos específicos, abordando temas que hacen a la enseñanza de diferentes áreas disciplinares en dichos niveles. Se espera con estas capacitaciones promover el desarrollo de competencias docentes, superando la formación inicial y promoviendo reflexión sobre la práctica.

En ocasión de que desde el Ministerio de Educación mencionado se establecieron nuevos lineamientos que sostienen un trabajo más integrado, problematizado y contextualizado a través de proyectos institucionales, se implementaron cursos que abordaron esta temática. El eje del trabajo fue el aprendizaje basado en proyectos (en adelante ABP). La novedad fue que en dichos cursos se tuvo grupos de gran heterogeneidad en cuanto a la formación de los docentes asistentes. Históricamente, y salvo excepciones, las propuestas habituales de capacitación estaban dirigidas a áreas específicas y a un único nivel educativo.

El trabajo que aquí se describe formó parte de una propuesta enmarcada en el curso "Herramientas para diseñar y guiar el aprendizaje basado en proyectos II" en uno de los CIIE de la provincia (distrito de San Miguel). Cabe aclarar que el dispositivo de capacitación se implementó con los docentes en servicio, antes del inicio del ciclo lectivo y tuvo alcance provincial a través de todos los CIIE de la jurisdicción.

\section{Aprendizaje basado en proyectos}

El ABP consiste en una propuesta metodológica que permite, como estrategia didáctica, que los participantes aborden alguna problemática de modo colaborativo integrando diferentes áreas de conocimiento. Es una forma de trabajo que ya cuenta con cierta antigüedad, pero genera mayor interés en los últimos tiempos (Domènech-Casal, 2016; Sanmartí, 2016). El ABP tiene un enfoque centrado en el alumno, pero requiere una importante participación del docente (Sánchez, 2013; Rodríguez-Arteche y Martínez-Aznar, 2016). De esta forma, los estudiantes son responsables de su proceso de aprendizaje y los docentes hacen de guía y son facilitadores de 
recursos (Johari y Bradshaw, 2008, Cascales, Carrillo y Redondo, 2017). Además, según Bell (2010), el enfoque promueve el aprendizaje social de los alumnos, brindándoles más posibilidades en relación con las competencias de comunicación propias del siglo XXI.

Existen otras posibilidades de diseño para el ABP, pero tomamos aquí el concepto de proyectos que contemplan elaborar un producto o resolver un problema, y ese concepto es el que da forma a muchas propuestas, según sostienen diversos autores (Larmer, Mergendoller y Boss, 2015). Un argumento a su favor, con el cual se tiene bastante acuerdo, es que los aprendizajes son más profundos y posibles de ser transferidos al realizarse de modo instrumental en la resolución de un problema donde los aprendices trabajando en comunidad tienen un papel activo (DomènechCasal, Lope y Mora, 2019). Pueden leerse estudios que abonan la conveniencia de integrar varias materias simultáneamente en un único proyecto (Gutiérrez-Pérez y Pirrami, 2011; Ayerbe López y Perales Palacios, 2020).

No obstante, el ABP es complejo de implementar, genera dificultades pedagógicas y resulta necesaria una esmerada planificación. Esto tiene como resultado una escasa incorporación de su metodología. Se tiene también cierta inercia de aquellas metodologías de carácter tradicional y la presión por cumplir con el currículo prescrito, lo que dificulta una mayor implantación del ABP (Ayerbe López y Perales Palacios, 2020). Implementar su metodología requiere un andamiaje que permita identificar los componentes didácticos que deben articularse en las propuestas de enseñanza (Domènech-Casal, 2016; Ertmer y Simons, 2005). Esto ha sido un eje transversal de nuestra propuesta de capacitación.

Por otro lado, un aspecto que suma dificultad es la evaluación en el contexto de los proyectos, particularmente de manera colegiada, lo que implica acuerdos entre docentes de distintas disciplinas. Considerando que existe tradición en la selección de criterios e instrumentos de evaluación de manera independiente, es un desafío evaluar en conjunto. Si bien quedará inscripta a condiciones institucionales, resulta adecuada la utilización de rúbricas para evaluar el proceso de enseñanza y aprendizaje centrado en proyectos. Estos instrumentos resaltan la potencialidad en el registro de criterios e indicadores que brindan información sobre el desempeño de los estudiantes. 


\section{Grupos heterogéneos de docentes aprendiendo sobre ABP}

Como se adelantó, el curso se desarrolló con docentes de diferentes áreas con el propósito de involucrarlos en un aprendizaje compartido que estimule especialmente el trabajo cooperativo en proyectos escolares que integren diferentes materias. Según Vilches y Gil (2011), el trabajo en grupos ofrece un papel orientador en cada participante, lo que ayuda a superar los errores personales y enriquecer los planteamientos individuales iniciales. Nuestra hipótesis de trabajo para la capacitación fue que al involucrar a los docentes en esta modalidad (la que además es imprescindible en el $\mathrm{ABP}$ ), estos adaptarían dichos formatos en su práctica propiciando mayores oportunidades de aprendizaje para sus alumnos.

Teniendo en cuenta el aprendizaje cooperativo, recuperamos el antiguo método de learning together de Johnson y Johnson (1995). Consideramos que el presente trabajo se adaptó a la metodología que plantea, ya que se conformaron grupos de hasta cinco integrantes y el objetivo era que todos alcanzaran dominio del tema de estudio presentado y ayudaran a sus compañeros en el aprendizaje. En el método la tarea es grupal: trabajan juntos para completar un material único (para el caso, un proyecto de ABP). Este material, además de ser la base para la evaluación del grupo, sirve a cada integrante para practicar, ayudarse, evaluarse a sí mismo y evaluar a sus compañeros. Esta línea coincide también con la propuesta de Perkins (2010), quien afirma que la mayoría de las veces las intervenciones formativas solo brindan un "telón de fondo informativo" en vez de una comprensión enriquecedora que potencie las capacidades de los estudiantes. Para ello propone la necesidad de participar en experiencias que generen situaciones de aprendizaje pleno, que los comprometa con su aprendizaje y dé una oportunidad de aprender de otros y con otros, favoreciendo la metacognición. Esta visión de Perkins es planteada en su libro en términos de un "juego completo" (2010, p. 29), haciendo un análogo con un deporte que invita a pensar las propuestas de enseñanza. Resultó adecuado introducirlo entre las lecturas indicadas a los docentes participantes.

Los diferentes trayectos formativos de los docentes debían relacionarse en un contexto de aprendizaje común donde tengan la oportunidad de contribuir con sus diferentes saberes y competencias. Según Coll, Mauri y Onrubia (2006) se demuestra en investigaciones psicoeducativas que en tales circunstancias (grupos de trabajo con la intervención de un docente guía, como fue este caso) se ponen en juego una serie de procesos interpsicológicos que otorgan significatividad y mayor sentido al aprendizaje. De esta forma, los entornos grupales promueven 
la colaboración entre los participantes: cada integrante es responsable de su propio aprendizaje, pero también del de los demás. En las interacciones, particularmente de un grupo heterogéneo, se ponen en juego estrategias lingüísticas y comunicacionales, así como estrategias de trabajo donde se utilizarían habilidades interpersonales que enriquecen el trabajo. Así, es el entorno social el que promueve competencias específicas (Perrenoud, 2004; Tejada Fernández, 2009; Gairín Sallan, 2011).

La colaboración entre pares, en este contexto, supone que los integrantes del grupo piensen cómo enfrentar los problemas y las dificultades que se les presentan. De algún modo, algunas de esas serían las futuras dificultades a las que se enfrentarían en el trabajo real en las escuelas de implementarse el ABP. Consideramos que atravesar esas situaciones en el curso tendría especial importancia en el desarrollo de competencias individuales y grupales, teniendo en cuenta que contábamos con personas de diferente formación y trayectorias.

En definitiva, el conocimiento es construido en un marco social determinado donde se despliega un proceso de interacción y compromiso que favorece un clima de cooperación y colaboración. Se asume que, en ausencia de fuertes restricciones personales, las prácticas de los docentes pueden evolucionar, con la condición de que esta evolución sea construida colectivamente en un trabajo cooperativo (Roditi, 2004).

\section{Descripción de la experiencia}

La propuesta de trabajo de 20 horas se desarrolló a través de tres encuentros presenciales de 4 horas y 8 no presenciales. Se implementó con un total de 56 docentes de diferentes áreas curriculares y niveles educativos (primario y secundario) - los cuales debían conformar libremente grupos de trabajo- y dos capacitadores. Para los encuentros se contempló emplear parte del tiempo en brindar explicaciones con apoyo gráfico en Power Point, lecturas grupales, puesta en común y trabajo en grupo. Los marcos teóricos fueron abordados en los tres encuentros, particularmente en el primero. El segundo y el tercer encuentro se centraron en el trabajo con modalidad de taller e intervenciones expositivas de los capacitadores en momentos específicos. En el segundo encuentro se presentó un ejemplo de proyecto, que se desarrolla en el presente artículo. Los grupos de docentes, conformados entre tres y cinco integrantes en general, estuvieron dedicados al diseño de un proyecto en particular. 
Para acreditar el curso los docentes debían concretar una producción grupal: un proyecto de trabajo que incorporara materias o áreas de incumbencia de los integrantes de los equipos. La asistencia necesaria estuvo establecida oficialmente en un 100\%. Además, cada docente debió presentar de modo individual y por escrito su postura, desde su especificidad formativa y área de desempeño, en relación con el ABP. Estas producciones fueron enviadas por correo electrónico días después de terminado el curso. Se realizaron las devoluciones correspondientes y en algunos casos se pidieron algunas reformulaciones para completar la aprobación.

\section{Objetivos del curso}

- Conocer las principales características del ABP.

- Analizar diversos tipos de productos como herramientas para visibilizar el aprendizaje de los alumnos.

- Caracterizar el rol de alumno y de los docentes en el aprendizaje basado en proyectos.

- Diseñar una propuesta de aprendizaje por proyecto que integre dos o más áreas disciplinares.

- Determinar criterios de evaluación y descriptores para el producto del proyecto ABP propuesto.

\section{Contenidos del curso}

- $\quad 1^{\circ}$ encuentro: Aspectos específicos del ABP. Su relación con los propósitos de la escuela. Problematización de contenidos. Rol del docente y rol del alumno. Fortalezas del trabajo en proyectos escolares integrando materias y contenidos.

- $\mathbf{2}^{\circ}$ encuentro: El diseño de proyectos, su escritura. La interdisciplinaridad en el aprendizaje. El trabajo formal en relación con los contenidos abordados en el ABP.

- $\quad 3^{\circ}$ encuentro: Evaluación de los aprendizajes de los estudiantes mediados por el trabajo en proyectos. Instrumentos (rúbricas, identificación de dimensiones, construcción de criterios, indicadores). Evaluación colegiada.

\section{Trabajo en las escuelas}

Los proyectos solicitados para la acreditación del curso debían contemplar un trabajo posible de implementar en las escuelas para la enseñanza de contenidos que atendiera a los 
lineamientos abordados en la capacitación. La consigna requería secuencias suficientemente articuladas donde se manifestaran las situaciones de enseñanza de los diferentes espacios, que deberían estar imbricadas claramente en un proyecto institucional común. Se presentaron formatos variados, aunque ajustados a un modelo de estructura que les fue brindado a los docentes.

\section{Ejemplos de planificación de proyectos de ABP}

Para que proyectos como los abordados en el curso puedan desarrollarse adecuadamente se requiere la articulación de espacios. Y, como la enseñanza transcurre en secuencias programadas por los docentes, resulta necesario vincularlas estratégicamente. Esto implica una progresión que se desarrolla con el avance de las clases. A los efectos de caracterizar posibles formatos en relación con el ordenamiento temporal de actividades, definimos como "sincrónico" al trabajo en paralelo dentro de una misma etapa (por ejemplo, dentro de una semana) y "asincrónico" cuando la vinculación de las materias con el proyecto se produce en diferentes momentos. Esto último podría resultar necesario, pongamos por caso, si los logros alcanzados en una materia sean requeridos con posterioridad en otra (el ejemplo particular presentado en la capacitación está diseñado de ese modo). Algunas de las posibilidades fueron presentadas gráficamente para su discusión (Figura 1).

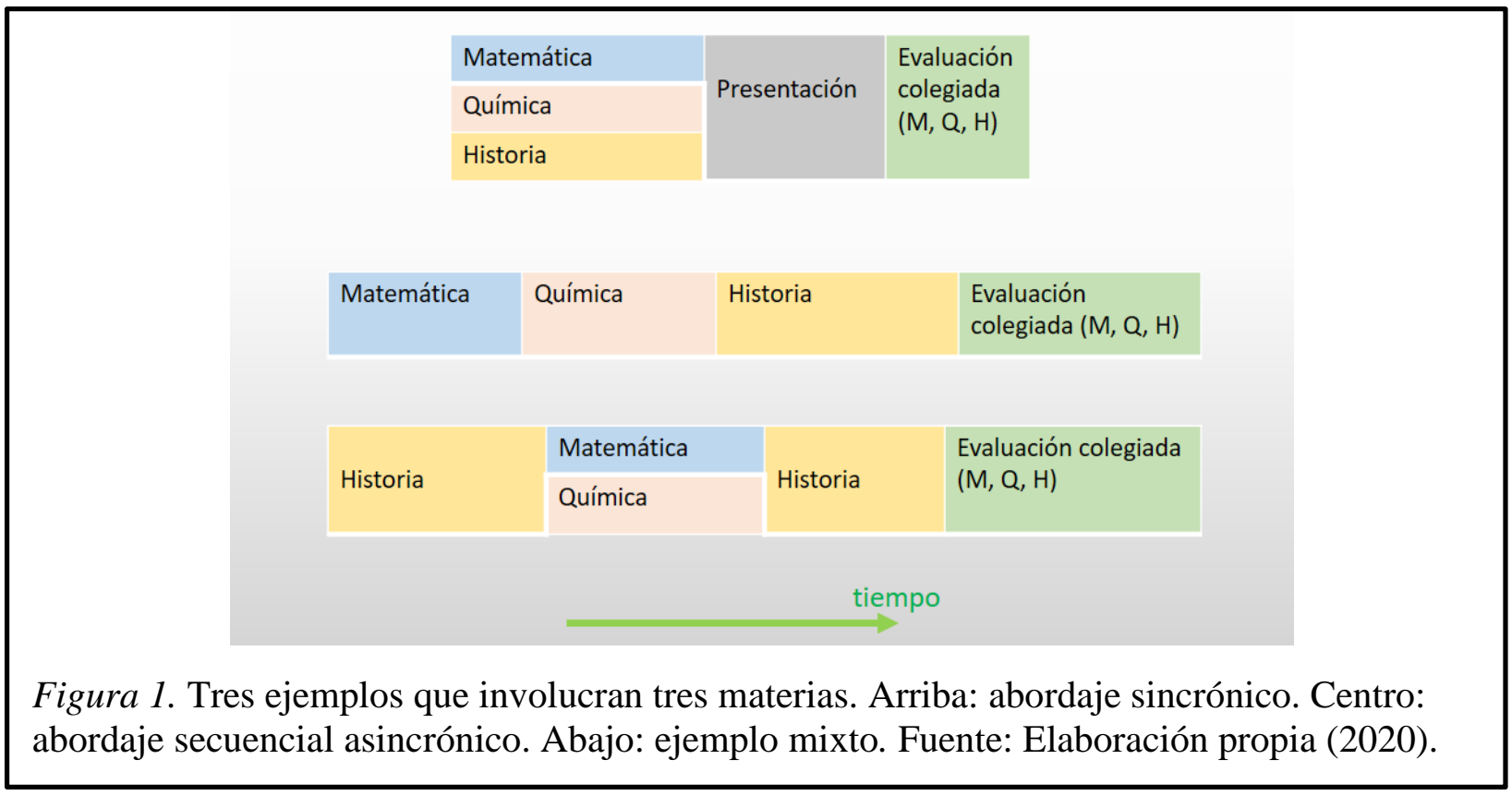


En la figura anterior puede verse además que la evaluación, que suele denominarse "colegiada", abarca un terreno común que requiere acuerdos docentes (no debe limitarse a una única posibilidad al final del trabajo).

\section{Ejemplo particular de ABP elaborado para la capacitación}

Asumiendo que la ejemplificación podría aportar más claridad al tema y entendiendo también que podría ser generadora de ideas y discusión, se elaboró un caso concreto de vinculación de materias de nivel secundario. No obstante, se consideró que este podía sesgar la mirada hacia un modelo cerrado de proyecto, lo cual no sería conveniente. Se hicieron las aclaraciones del caso para lograr la apertura a otros modelos posibles. El ejemplo (preparado para la ocasión, sin implementación real) se elaboró concibiéndolo para una escuela técnica. Consideramos que este tipo de instituciones cuentan con una fortaleza adicional que es el Taller ${ }^{1}$, donde se resaltan las actividades prácticas que podrían ser muy apropiadas en el ABP.

Se vincularon así cinco asignaturas del $4^{\circ}$ año: Física, Matemática, Geografía, Taller y Literatura. Se reconoce que en el ejemplo no se tiene "un problema" que reúna materias en el sentido típicamente concebido en el ABP. Sin embargo, se consideró oportuno ofrecer esta variante en la cual se tiene un tema estructurante que conforma un abordaje interdisciplinar. El nexo entre materias está fuertemente establecido (aspecto central en la capacitación) y permite una adecuada conceptualización de contenidos como un tratamiento no lineal del diseño curricular. Como un ejemplo modélico puede constituir un aporte, lo desarrollamos aquí con detalle.

Se tomó como caso de estudio desde las diferentes materias el muro Trombe. Este sistema físico es utilizado para calefaccionar viviendas con energía solar. Existen varios diseños, pero todos hacen uso de la convección del aire calentado por el sol, por lo que son amigables con el medio ambiente. En términos generales se trata de un gabinete cerrado, adosado a una pared de la vivienda y con una cubierta transparente donde ingresa la radiación solar. El ambiente de la vivienda que busca ser calefaccionado se comunica con el dispositivo a través de orificios por los cuales circula el aire. Sin entrar en mayores detalles técnicos de su construcción, se describe en adelante cómo fue utilizado en el ejemplo de proyecto (Figura 2).

\footnotetext{
${ }^{1}$ De forma simplificada denominamos "Taller" a lo que en realidad es un ámbito que reúne materias ligadas a diferentes actividades prácticas que responden a distintas especialidades y con sus nombres correspondientes.
} 


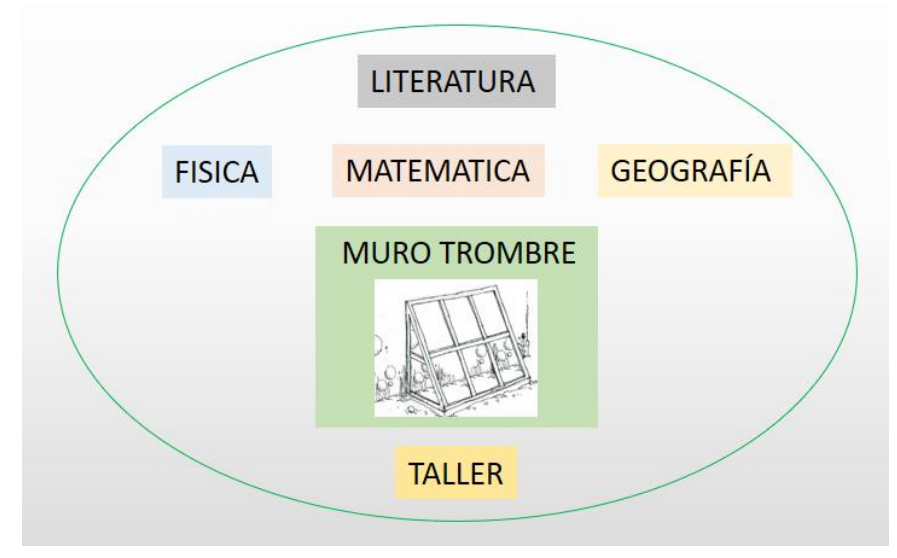

Figura 2. Esquema de la vinculación entre materias, donde el muro Trombe es el tema que permite el trabajo de contenidos. Fuente: Elaboración propia (2020)

En el ejemplo puede interpretarse que el dispositivo Trombe ${ }^{2}$ (DT) construido sea el "producto final" en los términos en que suele ser definido en el ABP (Figura 3). Si bien puede ser concebido de ese modo, cabe aclarar que otro significado del concepto, y quizá el más adecuado, sea que dicho producto es en realidad el informe final que los alumnos elaborarían. Vale decir también que el gabinete a construir opera como "módulo crítico", entendiendo así a los artefactos que en términos didácticos permiten abordar los procesos de la ciencia, de su naturaleza, y la tecnología en un contexto sociocultural (Cyrulies et al., 2012).
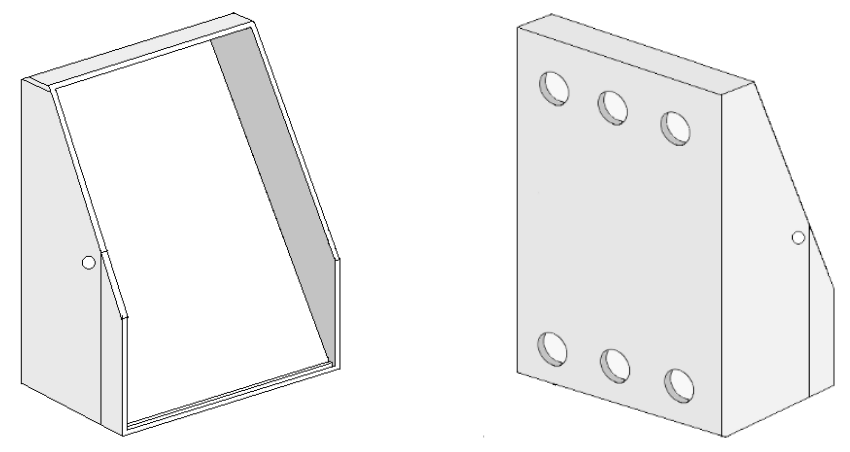

Figura 3. Prototipo de muro Trombe considerado en el marco del proyecto (gabinete experimental diseñado en Matemática, construido en Taller y utilizado en Física). Fuente: Elaboración propia (2020).

\footnotetext{
2 Decidimos denominar "dispositivo Trombre" al artefacto didáctico a construir en la escuela, diferenciándolo de
} "muro Trombre" que es la denominación genérica del sistema. 
Para el caso de Física, Matemática y Geografía se transcriben a continuación los contenidos extraídos del diseño curricular vigente de $4^{\circ}$ año con los que se vinculó el ejemplo. Se incluye en cada caso las orientaciones didácticas propuestas que entendemos se ajustan al ejemplo (se destacan en cursiva las que tienen especial relación) y seguidamente se describe el posible tratamiento de algunos de los contenidos citados. ${ }^{3}$ Para las materias Literatura y Taller se detallan más abajo las consideraciones del caso.

\section{FÍSICA}

\section{Ejes y núcleos de contenidos}

La energía en el mundo cotidiano

Diferentes formas de energía

Formas utilizables de la energía

Energías macroscópicas y su aprovechamiento

Energía hidroeléctrica. Energía eólica. Energía solar. Energía geotérmica. Energía mareomotriz. Energía nuclear. Aceleradores de partículas. Radioterapia.

"Este tema puede ser objeto de investigaciones escolares, a partir de problemas concretos surgidos de los estudiantes o propuestos por el docente, donde se puedan elaborar y poner a prueba hipótesis cualitativas acerca de las posibles variables de las que depende, tanto el intercambio de calor como la variación de temperatura de un objeto. [...] podrán hacerse gráficos a partir de datos para, comprobar o refutar las hipótesis en las investigaciones. A través de ecuaciones y análisis cualitativos puede estudiarse el intercambio de calor en diversas situaciones y proponer mecanismos para reforzar o atenuarlos [...] Una investigación interesante de bajo costo es el armado de una cocina solar, en la cual los tres mecanismos se conjugan y es posible producir un objeto concreto y analizar los factores que pueden optimizar su funcionamiento".

Para esta materia se consideró adecuado el uso del DT para el estudio de un caso concreto de aprovechamiento de energías renovables. Y, particularmente, resulta un buen ejemplo de los efectos de la convección, considerando que esta tiene una formulación demasiado compleja para abordar en secundaria. El DT construido en Taller sería de gran utilidad en prácticas experimentales que permitirían la toma de datos y el análisis de resultados a procesar con los alumnos. Se consideró de utilidad el uso de un simulador gratuito de gran potencialidad de modelización de la transmisión de calor en diferentes casos (Energy2D, de descarga gratuita) que permite modelizar el movimiento del aire por convección en una configuración de muro Trombe.

\footnotetext{
${ }^{3}$ Por razones de extensión solo se dan visiones generales que consideramos suficientes para el ejemplo, tal como se presentaron en el curso.
} 


\section{MATEMÁTICA}

Eje geometría y álgebra: núcleos sintéticos de contenidos

Resolución de problemas que involucren figuras planas y cuerpos tridimensionales_que relacionen conceptos trabajados con anterioridad integrados con los nuevos que se aborden: teorema de Thales, trigonometría, teoremas del seno y del coseno.

Semejanza de figuras planas

Teorema de Thales

Trigonometría

Lugar geométrico

Parábola

“Se profundizará en la resolución de ecuaciones e inecuaciones analizando formas gráficas y analíticas. Se modelizarán y resolverán situaciones intra y extra-matemáticas mediante ecuaciones e inecuaciones”.

Para Matemática el proyecto puede ofrecer un contexto que permita una problematización de algunos contenidos, particularmente de la geometría. Tal vez más interesante resulta si una matemática aplicada permite resolver problemas reales. El desarrollo del DT incluye rectángulos y triángulos rectángulos a ser cortados de una tabla. Eso amerita optimizar los cortes lo que implica resolverlo en forma algebraica (Figura 4).
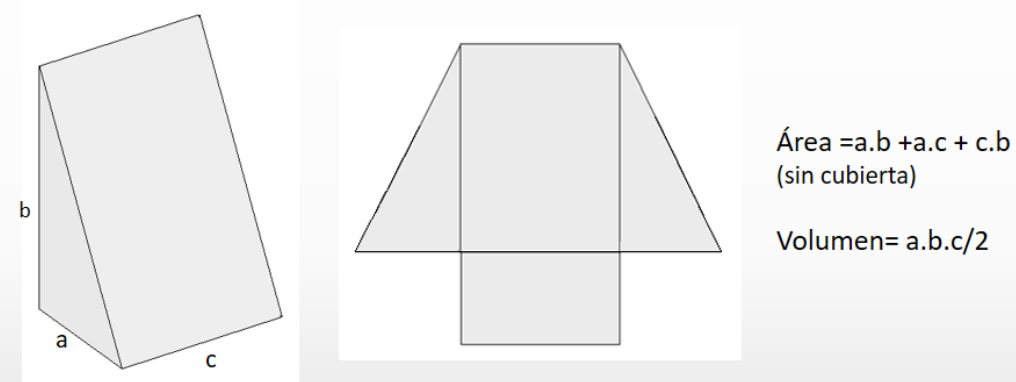

Figura 4. Desarrollo de la primera etapa del DT y su traducción en lenguaje matemático. Fuente: Elaboración propia (2020).

Las proporciones de las caras triangulares estarán dadas por la inclinación de la cubierta transparente, la que resulta adecuada tomando como referencia la declinación variable del sol a lo largo de año. Aquellas, naturalmente, pueden ser determinadas a través de la trigonometría (Figura 5). Por otra parte, el volumen encerrado dependerá también de la inclinación y podría considerarse que, a mayor volumen, menor calentamiento del aire, lo que no es recomendable en el dispositivo. 
Debido al elevado grado de complejidad del problema, será el docente quien plantee un modelo sencillo abordable en la clase de matemática, que permita definir un diseño adecuado del DT para ser construido en el Taller.
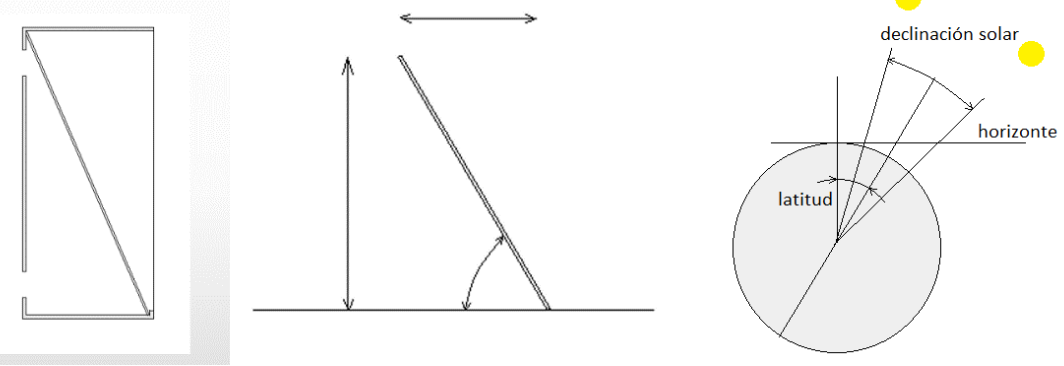

Figura 5. La inclinación óptima de la cubierta define las medidas de las tablas. Un problema para abordar con los alumnos es elegir la inclinación adecuada atendiendo a la posición del sol. Fuente: Elaboración propia (2020).

Otra problematización posible es el análisis de la relación perímetro-área de diferentes figuras geométricas en un ejemplo concreto, que en este caso sería caracterizar a los orificios necesarios para la convección. Aun reconociendo que los orificios circulares son los que resultan más fáciles de realizar si se hace uso de máquinas-herramientas, no es tan conocido que además es la forma que minimiza el perímetro respecto del área encerrada. Esto, que también hace mínimo el corte de material, puede ser demostrado en términos matemáticos planteando las ecuaciones correspondientes (Figura 6).

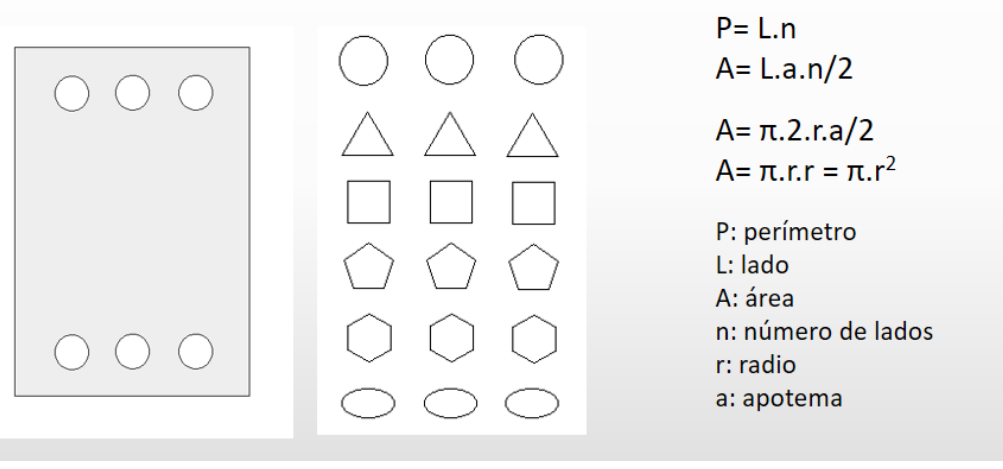

Figura 6. El problema de los orificios en Matemática. Frente a todas las formas posibles, la circular es la óptima. Fuente: Elaboración propia (2020). 


\section{GEOGRAFÍA}

\section{Unidades de contenidos y objetos de estudio}

Las actuales condiciones económico-políticas del desarrollo desigual mundial La desigual distribución mundial de los recursos. Problemas ambientales y geopolíticos asociados La distribución de la población mundial y sus transformaciones en el actual contexto de la globalización neoliberal

Las transformaciones urbanas y rurales en el contexto de la globalización neoliberal

Reconocimiento de la diferencia cultural, la identidad y la consideración del otro y de otros espacios.

Objetivos:

Conocer información y explicaciones sobre las relaciones de desigualdad que históricamente se han estructurado en torno al comercio internacional y/o al intercambio comercial, entre países centrales y periféricos y de qué manera esto influye en las condiciones de vida de una parte importante de la población, por ejemplo, en la de los campesinos de los países menos desarrollados.

La Geografía, actualmente enseñada como ciencia social, tiene una gran cuota de sensibilización frente a los problemas sociales, tal como puede interpretarse del recuadro anterior. Teniendo en cuenta esto, se eligió un caso de estudio que incluye la utilización de un muro Trombe en una comunidad de los andes peruanos cuya vida transcurre en condiciones muy extremas.

El trabajo escolar a partir de la lectura del caso, tomado del diario español El País, puede resultar de gran valor para los propósitos de la materia. Se presenta a continuación el texto de la nota (Cuadro 1). El planteo quedó liberado a las consignas que se crean pertinentes. 
Cuadro 1

Propuesta de lectura para Geografía

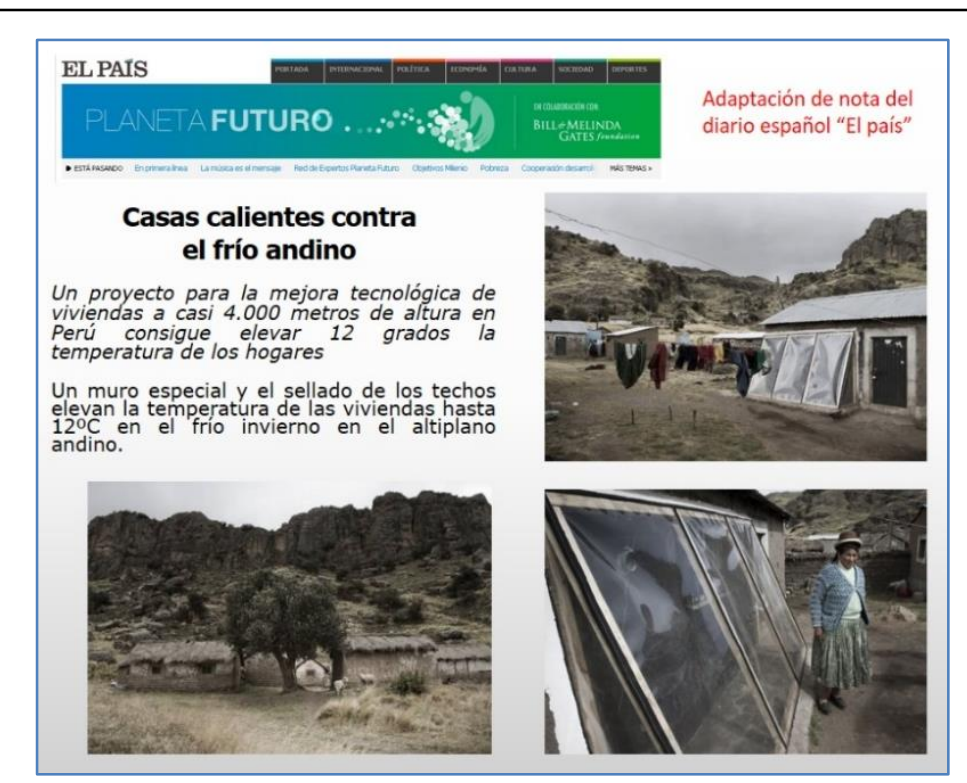

"Acá el sol es bien fuerte. No calienta, quema", comenta Nancy mientras conduce por una carretera circundante al lago Titicaca. Esta bióloga hace todos los días el recorrido que la lleva desde el sureste de Perú hasta los diferentes emplazamientos de la organización Kusimayo. Durante el tiempo que ella y Joaquín trabajaron juntos en una granja, se dieron cuenta de las carencias de este rincón del país. Poco después se crea una organización sin fines de lucro que desde el año 2008 desarrolla proyectos para mejorar las condiciones de vida en Perú. La población rural alto andina pasa por ser la más pobre y excluida del país. Las bajas temperaturas provocan que haya una elevada tasa de casos de infecciones respiratorias agudas, la principal causa de muerte en niños en zonas rurales. Los hogares de muchas comunidades campesinas son incapaces de atenuar los estragos que derivan de las gélidas temperaturas. En la mayoría de casos las viviendas no cuentan con la infraestructura mínima para evitar el paso del frío al interior de la misma, como puede ser el sellado de las puertas, ventanas y techo, que suele ser muy deficitario. A esta problemática se suma el escaso acceso a la energía. La gran mayoría de hogares cuenta con cocinas de leña o bosta y se hace un uso ineficiente y contaminante de los combustibles. Nancy, Joaquín y Laura se desmarcan de las acciones solidarias paliativas que realiza el Estado. El resultado de las labores en este sentido por parte del gobierno es, en ocasiones, inefectivo por la falta de recursos y la manera de hacer llegar la ropa y los alimentos.

La comunidad de Rivera Coylata se intuye vacía. Se escucha el sonido de las hojas al moverse, el cacareo de las gallinas y el silencio. Mucho silencio. Al forastero la respiración se le complica y el oxígeno parece escasear. Kusimayo ha antepuesto la entrega de ropa de abrigo por la de implementar soluciones, como la del paquete tecnológico Casa Caliente Limpia desarrollado por el Grupo de Apoyo Rural de la Universidad Católica del Perú, el cual pretende modificar las casas mediante la instalación de paredes calientes, el sellado de los techos y cocinas mejoradas para evitar el humo en su interior.

La temperatura en invierno en esta región alcanza los 18 grados bajo cero. En el interior de los hogares, antes de la implantación de las Casas Calientes, la temperatura era de -5 aproximadamente. Ahora se mantiene en torno a los siete grados. La directiva de la comunidad y la población están comprometidas con la implementación del paquete tecnológico. La Comunidad Rivera Coylata aporta la mano de obra y los materiales: adobe, piedras. La madera y el policarbonato vienen de afuera.

El interior del Muro Trombe está relleno de piedras de río de color negro para conseguir un mejor almacenamiento de calor durante el día. Este sistema consiste en un recinto adyacente a la casa que tiene una pared expuesta al sol. Los agujeros puestos específicamente en la pared del muro Trombe (en la parte inferior y superior) permiten que el aire calentado por el sol circule en la casa, a su vez el aire frío vuelve a entrar al recinto expuesto al sol, proporcionando calefacción a la casa. Para el éxito de este implemento es importante aislar el techo con arpillera, un material local que tiene la función de retener el calor transferido por el Muro Trombe en el interior de la estancia.

Fuente: https://elpais.com/elpais/2014/06/25/planeta_futuro/1403689551_322622.html 


\section{LITERATURA Y TALLER}

Finalmente, la materia Literatura aporta de modo transversal en la etapa final, en la que se espera un informe escrito por parte de los alumnos vinculando lo trabajado en los distintos espacios. Cabe señalar que en las materias elegidas (y también en otras) hay referencias explícitas a la importancia de la lectura y la escritura con las especificidades de cada espacio. En el Taller se diseña el DT que será utilizado en Física, en función de las determinaciones obtenidas en Matemática, y se construye esencialmente por medio de operaciones de carpintería.

\section{Presentación temporal de las actividades}

Las propuestas se relacionan entre sí a lo largo de 8 semanas antes de la evaluación final colegiada. El proceso, tal como fue diseñado, se muestra en el Cuadro 2. Las flechas indican cuando las relaciones entre materias conllevan resultados de una que son necesarios en otra.

Cuadro 2

Cronograma de actividades de las materias

\begin{tabular}{|c|c|c|c|c|c|}
\hline ASIGN/SEM & 1 & 2 & 3 & 4 & 5 \\
\hline FÍSICA & $\begin{array}{l}\text { Conceptuali- } \\
\text { zación de } \\
\text { Transmisión } \\
\text { de calor }\end{array}$ & $\begin{array}{l}\text { MT como artefacto } \\
\text { para el } \\
\text { aprovechamiento de } \\
\text { la energía solar }\end{array}$ & $\begin{array}{l}\text { Simulación con } \\
\text { Energy } 2 D\end{array}$ & & $\begin{array}{l}\text { Utilización en } \\
\text { laboratorio } \\
\text { para } \\
\text { mediciones y } \\
\text { levantamien- } \\
\text { to de curvas }\end{array}$ \\
\hline $\begin{array}{l}\text { MATEMÁ- } \\
\text { TICA }\end{array}$ & $\begin{array}{l}\text { Recuperación } \\
\text { de saberes } \\
\text { sobre figuras }\end{array}$ & $\begin{array}{l}\text { Recuperación saberes } \\
\text { s/cuerpos. } \\
\text { Problematización } \\
\text { sobre figuras y } \\
\text { cuerpos }\end{array}$ & $\begin{array}{l}\text { DT: Determinación } \\
\text { de formas } \\
\text { óptimas. Cálculo } \\
\text { de volumen. Uso } \\
\text { de trigonometría }\end{array}$ & & \\
\hline GEOGRAFÍA & $\begin{array}{l}\text { Distribución } \\
\text { de la } \\
\text { población } \\
\text { mundial }\end{array}$ & $\begin{array}{l}\text { Condiciones } \\
\text { económico-políticas } \\
\text { del desarrollo } \\
\text { desigual mundial. } \\
\text { Caso M Ten Perú }\end{array}$ & & & \\
\hline TALLER & & & $\begin{array}{l}\text { Diseño e inicio de } \\
\text { construcción de } \\
\text { DT a escala }\end{array}$ & Construcción & \\
\hline
\end{tabular}

\begin{tabular}{|c|c|c|c|c|}
\hline & 6 & 7 & 8 & 9 \\
\hline FISICA & $\begin{array}{l}\text { Escritura (leer y escribir en } \\
\text { ciencias) }\end{array}$ & \multirow{5}{*}{$\begin{array}{l}\text { Producción de un } \\
\text { informe } \\
\text { grupal con los } \\
\text { escritos } \\
\text { disciplinares }\end{array}$} & \multirow{5}{*}{$\begin{array}{l}\text { Comunicación del } \\
\text { trabajo a otros } \\
\text { cursos/ familia } \\
\text { Feria de ciencias }\end{array}$} & \multirow{5}{*}{$\begin{array}{l}\text { Evaluación } \\
\text { colegiada }\end{array}$} \\
\hline MATEMÁTICA & $\begin{array}{l}\text { Escritura (leer y escribir en } \\
\text { matemática) }\end{array}$ & & & \\
\hline GEOGRAFÍA & $\begin{array}{l}\text { Escritura (la enseñanza de la G a } \\
\text { través de la lectura, la escritura } \\
\text { y otros lenguajes de tradición } \\
\text { disciplinar) }\end{array}$ & & & \\
\hline \multirow[t]{2}{*}{ TALLER } & Elaboración de planos & & & \\
\hline & LITERATURA (ароуо) & & & \\
\hline
\end{tabular}


El ejemplo descrito fue desarrollado en el primer encuentro de la capacitación, y sirvió de soporte para su análisis tomando en cuenta los marcos teóricos abordados. En los encuentros siguientes se propuso una dinámica con mayor producción por parte de los asistentes.

\section{Aplicación de una encuesta}

Al finalizar la capacitación se realizó una encuesta individual (Cuadro 3) para indagar sobre las representaciones de los docentes y su evaluación del curso.

Cuadro 3

Encuesta aplicada a los 56 docentes participantes

\section{ENCUESTA}

Nombre y apellido:

Por favor, indique cual es el área/ materia en la que se desempeña:

A) Sobre las propuestas escolares que integren materias en un trabajo común basado en ABP se pide responder los siguientes puntos:

En relación al aprendizaje de contenidos por parte de los chicos a través de una propuesta integradora como la citada:

1- ¿Qué ventajas identifica?

2- ¿Qué dificultades identifica?

En cuanto a su práctica docente desarrollando trabajo en equipo con colegas de otras áreas:

3- ¿Qué reconoce como positivo?

4- ¿Y cómo negativo?

B) Opinión libre sobre la presente capacitación (aportes, materiales, etc.):

\section{Resultados de la capacitación}

Los docentes participaron activamente en las actividades propuestas en los tres encuentros. Se considera que el planteo de Vilches y Gil (2011) en relación con el papel orientador sobre cada participante efectivamente se produjo en los intercambios al interior de los grupos. Nuestras intervenciones fueron permanentes y, según el análisis tomado de Coll et al. (2006), dieron particular sentido al aprendizaje de los colegas. Dentro de esta dinámica, el diseño de los proyectos fue discutido en tiempos presenciales y su elaboración final se completó fuera de horario. 
Finalmente, los proyectos fueron enviados por correo electrónico y luego de su evaluación se les brindó una devolución.

Los docentes conformaron 14 grupos heterogéneos y cada uno produjo un proyecto. Se obtuvo una gran variedad de temas, destacándose la elección de problemáticas que connotan sensibilidad por aspectos sociales (pueblos originarios), atienden a una construcción de ciudadanía (educación vial, ESI), propuestas culturales (arte, folclore) o cuidado del ambiente (reutilización de envases). ${ }^{4}$ Las materias involucradas abarcaron a diferentes áreas del conocimiento: ciencias sociales, naturales y exactas, inclusive se tuvo participación de bibliotecarios. Naturalmente, con diferente presencia según el grupo.

Las secuencias de trabajo prácticamente en todos los casos presentaban, implícitamente, un abordaje sincrónico de las materias. Este formato hizo que, en términos relativos, sean escasas las actividades de interrelación de etapas de algún modo similar al ejemplo del muro Trombe. Prevaleció un esquema en el cual cada materia aportaría, desde una secuenciación de actividades propia, a conformar un "producto final" (comunicación a la comunidad, mural, construcción con material concreto, etc.). No obstante, dicho producto final tuvo, en muchos casos, una marcada relación con el ejemplo trabajado, en términos de una propuesta de escritura final en la implementación del proyecto.

Podemos afirmar que todos los temas abordados efectivamente son apropiados y recomendables para ser trabajados en las escuelas. Esto muestra que se logran consensos en la elección de temas estructurantes para el ABP por parte de los docentes. Los propósitos generales de los proyectos terminaron siendo diferentes según los grupos, lo que era algo esperado. En varios casos, a los docentes los movilizó cierta problemática específica de alguna escuela de origen.

Entre las dificultades encontradas en las producciones resalta una que debería ser tenida en cuenta para próximas instancias: el tratamiento específico de los contenidos en los proyectos. En este sentido, resultó bastante recurrente el trabajo con conceptos propios de cada materia, pero de un modo algo difuso en cuanto a su aprendizaje en términos formales. Es decir, nos encontramos con poca explicitación sobre la conceptualización de contenidos tomando con contexto de trabajo al proyecto. De alguna manera, se trabajaba con contenidos, pero "aplicándolos" a diferentes situaciones, casi asumiendo que ya están aprendidos. Esto es precisamente lo que Larmer y

\footnotetext{
${ }^{4}$ Esta clasificación no es absoluta, las categorías pueden interrelacionarse según el criterio considerado. Aquí solo la usamos para una primera diferenciación.
} 
Mergendoller (2010) identifican como el colofón de una determinada unidad didáctica, si el proyecto no es el eje vertebrador. Esta visión aplicativa, si bien es factible, no es la línea de trabajo sugerida en el curso. Un desafío mayor consiste en que el escenario del proyecto sea el que establece la necesidad de aprender los contenidos formales. Entendemos que si no se plantea de ese modo puede atentar contra un adecuado cumplimiento de los programas de las asignaturas. También dentro de los proyectos realizados hubo algunos en los que las actividades propuestas se ajustarían más para etapas de escolarización anteriores a las de la implementación del proyecto, al menos en lo planteado en ciertas materias.

Se detectaron ciertos problemas de coherencia interna en determinados casos. Uno, bastante observado, fue el planteo de objetivos de aprendizaje ambiciosos. Aunque a partir de su lectura aislada no genera ninguna idea de imposibilidad, no parecerían alcanzarse con las propuestas. A modo de ejemplo se trascriben algunos.

"Realicen informes científicos e históricos para el abordaje de nuevas áreas de estudio".

"Problematizar la utilización de estereotipos y clichés en la construcción y elaboración de trabajos de producción".

También se identificaron casos con cierta vaguedad en su enunciación:

"Generar actividades que impacten en lo cotidiano y en la comunidad educativa".

"Puedan apropiarse de los diferentes contenidos y establecer relación entre ellos acomodándolos a sus saberes previos".

En algunos pocos casos los objetivos son dudosos en cuanto a su pertinencia:

“Que los alumnos conozcan el límite de su autonomía".

Entendemos que la devolución que se envió sobre estas cuestiones pudo aportar a un planteo más realista o concreto en función de las actividades programadas. Se propuso como estrategia recorrer nuevamente lo planificado en el proyecto y "volver" a los objetivos para ajustarlos adecuadamente. Después de todo, es lo propuesto a través de las actividades lo que da sentido a los objetivos.

Otra característica identificada en ciertas producciones fue una descripción escasa de las actividades para los estudiantes, que se planteaban en términos mucho más generales. Dicho de otro modo, resultaba dificultoso, en esos casos, construirse una escena de lo que realizarían los 
alumnos y el docente en el desarrollo de la secuencia. Es posible que algún formato de planificaciones anuales utilizadas en algunas instituciones, escritas con cierto grado de generalidad, haya operado de modelo en este sentido. Estos son algunos ejemplos:

"Se les pide a los alumnos que hagan estadísticas con las encuestas realizadas por todos los grupos y que se grafique".

"Se les solicita a los estudiantes que profundicen sus conocimientos sobre la contaminación y el reciclado de plásticos [...]”.

En cuanto a las actividades del docente, se pudo observar que en general están menos explicitadas que las actividades de los alumnos. Si bien se expresan en términos amplios dentro de las clases, fue algo muy común la escasa descripción de las acciones específicas dentro de la secuencia de trabajo. En algunos casos resultaron algo imprecisas:

"Brindar conceptualizaciones para la comprensión de la estructura dramática y sus componentes, como instrumento de análisis de la producción realizada".

"Se pedirá que realicen un boceto que pueda integrar los conocimientos, y reflexiones sobre el tema "el trabajo" que incluya saberes de las 4 materias que intervienen en el proyecto".

Sobre las rúbricas requeridas, pudo observarse en general una producción ajustada, posiblemente favorecida por una mayor intervención conjunta de los integrantes en este caso al solicitárseles un único instrumento compartido y, a la vez, adecuado a cada espacio curricular.

Por otro lado, el procesamiento de los resultados de la encuesta se presenta en las tablas 1 y $2 .^{5}$ Allí se agrupan las respuestas a las preguntas 1 - 2 y 3 - 4 respectivamente, las que permiten obtener algunos datos sobre la implementación del ABP según lo interpretan los propios docentes. No realizamos ninguna operacionalización sobre el punto B de la encuesta. Cabe señalar, sin embargo, que en todos los casos pudieron leerse comentarios positivos en dicho punto.

\footnotetext{
${ }^{5}$ Se aclara que la suma de las respuestas supera a la cantidad de participantes. Esto ocurre porque las respuestas de algunos incluyeron más de una de las categorías que hemos considerado para el análisis.
} 
Tabla 1

Resultados de la encuesta en relación con el aprendizaje de los alumnos (A)

\begin{tabular}{|c|c|c|c|c|c|}
\hline \multicolumn{6}{|c|}{ En relación al aprendizaje de contenidos por parte de los chicos } \\
\hline \multicolumn{3}{|c|}{ ¿Qué ventajas identifica? } & \multicolumn{3}{|c|}{ ¿Qué dificultades identifica? } \\
\hline Categoría & Cantidad & Porcentaje & Categoría & Cantidad & Porcentaje \\
\hline trabajo cooperativo/colaborativo & 15 & 26,8 & falta de tiempo de los docentes & 10 & 17,9 \\
\hline los A reconocen relaciones entre áreas & 13 & 23,2 & dificultades en coordinar/consensuar & 10 & 17,9 \\
\hline los A son protagonistas y/o activos & 13 & 23,2 & mayor necesidad de compromiso del A & 9 & 16,1 \\
\hline produce mayor comprensión & 9 & 16,1 & no responde/ respuesta poco clara & 6 & 10,7 \\
\hline genera autonomía & 8 & 14,3 & escuelas sin las condiciones adecuadas & 5 & 8,9 \\
\hline los A descubren relaciones con el mundo & 7 & 12,5 & tiempo que demanda desarrollarlo & 4 & 7,1 \\
\hline atiende a intereses & 7 & 12,5 & dificultades en la evaluación & 3 & 5,4 \\
\hline motiva & 6 & 10,7 & no reconoce nada negativo & 3 & 5,4 \\
\hline se resuelven problemas relevantes & 5 & 8,9 & más trabajo/ dificultades por parte del A & 2 & 3,6 \\
\hline favorece la metacognición & 4 & 7,1 & dificultades para alumnos solitarios & 2 & 3,6 \\
\hline desarrolla competencias/ habilidades & 4 & 7,1 & dificultad en hallar temática adecuada & 2 & 3,6 \\
\hline desarrolla pensamiento crítico & 4 & 7,1 & requiere cambio de perspectiva & 1 & 1,8 \\
\hline se comparte responsabilidades & 3 & 5,4 & A destacados prefieren trabajar solos & 1 & 1,8 \\
\hline genera autocrítica & 2 & 3,6 & & & \\
\hline otorga más sentido a los contenidos & 2 & 3,6 & & & \\
\hline poco claro o no responde lo solicitado & 2 & 3,6 & & & \\
\hline aprendizaje menos aburrido & 1 & 1,8 & & & \\
\hline genera creatividad & 1 & 1,8 & & & \\
\hline se crean nuevos interrogantes & 1 & 1,8 & & & \\
\hline permite aplicar metodología científica & 1 & 1,8 & & & \\
\hline
\end{tabular}

\section{Fuente: Elaboración propia (2020)}


Tabla 2

Resultados de la encuesta en relación con la práctica docente

\begin{tabular}{|c|c|c|c|c|c|}
\hline \multicolumn{6}{|c|}{ En cuanto a su práctica docente desarrollando trabajo en equipo con colegas de otras áreas } \\
\hline \multicolumn{3}{|c|}{ ¿Qué reconoce como positivo? } & \multicolumn{3}{|c|}{ ¿Y cómo negativo? } \\
\hline Categoría & Cantidad & Porcentaje & Categoría & Cantidad & Porcentaje \\
\hline enriquecimiento de los docentes & 15 & 26,8 & dificultad en coordinar horarios/lugares & 13 & 23,2 \\
\hline se comparten puntos de vista & 8 & 14,3 & falta de tiempo de docentes & 13 & 23,2 \\
\hline aumenta la interacción entre colegas & 7 & 12,5 & docentes estructurados & 8 & 14,3 \\
\hline los docentes se involucran en otras materias & 6 & 10,7 & lograr acuerdos entre docentes & 5 & 8,9 \\
\hline entusiasmo docente se traslada a los A & 5 & 8,9 & falta de recursos & 5 & 8,9 \\
\hline fortalece las prácticas pedagógicas & 4 & 7,1 & no reconoce nada negativo & 5 & 8,9 \\
\hline no responde/ respuesta poco clara & 4 & 7,1 & esfuerzo extra de los docentes & 4 & 7,1 \\
\hline genera cooperativismo y/ empatía & 3 & 5,4 & no responde/ respuesta poco clara & 3 & 5,4 \\
\hline generar acuerdos en la evaluación & 3 & 5,4 & dificultad para encontrar el tema & 2 & 3,6 \\
\hline fomenta la creatividad docente & 1 & 1,8 & se diluye la responsabilidad de docentes & 2 & 3,6 \\
\hline permite valorar el trabajo ajeno & 1 & 1,8 & poco acompañamiento institucional & 1 & 1,8 \\
\hline se evita una enseñanza atomizada & 1 & 1,8 & & & \\
\hline
\end{tabular}

Fuente: Elaboración propia (2020)

A partir de las categorías construidas puede verse que el $\mathrm{ABP}$ es altamente valorado por los docentes en diferentes dimensiones. En relación con el aprendizaje se destaca la mayor participación grupal de los alumnos en problemas genuinos de su interés. También se señala especialmente el protagonismo estudiantil y una expectativa de mayor comprensión. Sin embargo, expresan ciertos problemas para su implementación, marcando particularmente la falta de tiempo junto a la complicación de lograr una adecuada coordinación y alcanzar acuerdos. De todos modos, son mínimas las observaciones dirigidas a la identificación de dificultades en relación con el aprendizaje, a excepción de las 9 respuestas $(16,1 \%)$ que refieren al mayor compromiso de los alumnos (y esto, según se interprete, también podría ser considerado una ventaja).

Estas manifestaciones concuerdan con lo expresado en trabajos previos (Davini, 2008; Ayerbe López y Perales Palacios, 2020) en donde se afirma que son las formas rígidas de organización y del trabajo de los docentes las que tienden a ser un obstáculo en el desarrollo de proyectos de acción integrados al requerir cambios en su rol. Es interesante observar que algunas respuestas sobre las dificultades en el aprendizaje de contenidos están en realidad más vinculadas 
con el propio quehacer docente en relación con la institución que con los procesos cognitivos de los estudiantes. Evidentemente es en aquel escenario donde ven los obstáculos.

La Tabla 2 muestra un interesante hallazgo y es que muchos docentes se ven enriquecidos ellos mismos con la implementación del ABP. Posiblemente esto pueda cruzarse particularmente con las categorías que refieren a compartir puntos de vista entre colegas, aumentar su interacción y ampliar la mirada a otras áreas de conocimiento que son donde se hallan la mayoría de las respuestas. Encontramos sintonía con las afirmaciones de Roditi (2004) si a esto lo interpretamos como una evolución en el colectivo docente sustentada en un trabajo cooperativo.

En contrapartida, nuevamente aparece aquí, como aspecto negativo, la falta de tiempo y dificultades en poder lograr acuerdos. En relación con esto último concebimos una posible relación con la calificación de "colegas estructurados" en términos de los propios participantes. Quizá sea de resaltar que la totalidad de las respuestas que lo afirman (8 que representan el 14,3\%) califican de estructurados a otros docentes, aunque ninguno hace referencia a esa posible característica en sí mismo.

No deja de ser llamativo detectar que, aunque las respuestas dejan ver la conveniencia de integrar áreas por diferentes razones, solo una respuesta hace referencia explícita a la superación de una enseñanza "atomizada". Nuestra hipótesis es que dicho concepto, propio del ABP, resultaba obvio para los participantes y no merecía ser mencionado en la encuesta (no resultaría una conclusión, fue un argumento inicial de la capacitación).
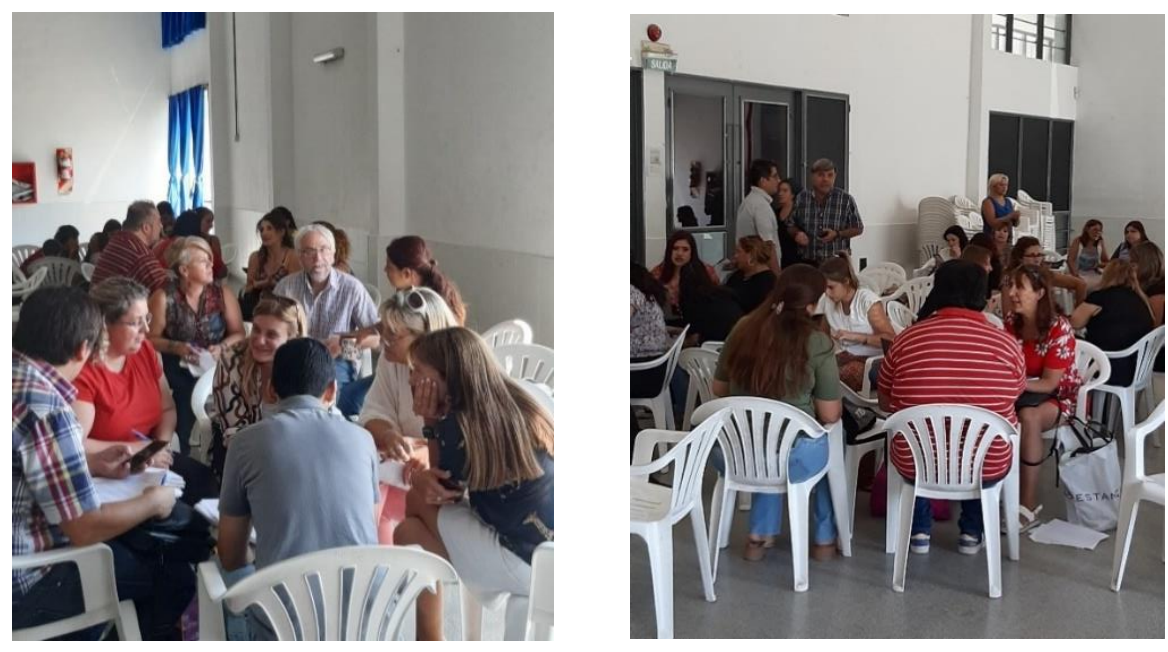

Figura 7. Escenas del trabajo con los docentes. Fuente: Elaboración propia (2020). 


\section{Discusión y conclusiones}

La propuesta permitió comprobar la viabilidad de un trabajo conjunto de grupos heterogéneos conformados por integrantes de áreas/disciplinas educativas diferentes. Entendemos que plantear el aprendizaje en dicha heterogeneidad ha inducido un espacio de reflexión y discusión sobre la práctica docente de un modo no habitual en las escuelas. Un aspecto puntualmente discutido con los docentes fue la consideración de diferentes posibilidades de una enseñanza contextualizada de contenidos, superando un formato institucional quizá dominante. Muchos participantes comunicaron que nunca habían trabajado en equipos heterogéneos cursando una capacitación ni tampoco concibiendo propuestas didácticas en la escuela con la integración planteada en el ABP. Es conocida, y fue admitida por varios cursantes, la escasa vinculación que habitualmente se tiene en las asignaturas de la escuela secundaria. El ABP puede modificar favorablemente este escenario común y en esto se tuvo consenso.

Un detalle que resaltamos y que se desprende tanto de las encuestas como de los intercambios personales es la gran predisposición de los docentes en la propuesta de capacitación y en la valoración positiva que han hecho del ABP. Todos los participantes, sin excepción, se manifestaron en su favor. Identificaron dificultades en su ejecución, pero no manifestaron preferir otros modos de enseñanza. Nos parece importante destacar esto ya que, si se cuenta con esta aceptación en el colectivo docente, deberá facilitarse el apoyo formativo y las condiciones institucionales para que efectivamente el ABP tenga un mayor grado de implementación. En nuestros intercambios personales con los docentes se tuvo particular consideración por el ejemplo planteado desde la capacitación. Al respecto lo valoraron como positivo al dar cuenta de un posible abordaje de contenidos integrados en un proyecto.

Por otra parte, si bien la duración del curso estuvo establecida por regulaciones oficiales, en nuestra opinión los tiempos para poder desarrollar esta propuesta de curso ABP resultaron acotados. Sobre todo, por el tiempo que demanda el proceso de elaboración de los proyectos si se espera sostener un debido acompañamiento desde una capacitación. Así lo manifestaron también varios docentes. Acordamos con Sánchez (2013) en que la metodología claramente tiene particulares dificultades, sobre todo en su iniciación. Uno de los mayores desafíos, y del cual no cabe esperar una inmediata incorporación a la práctica por la apropiación que requiere, parece ser tomar al proyecto como eje vertebrador y no como colofón, según aquellas palabras de Larmer y Mergendoller (2010). 
Entendemos que los aspectos señalados aquí justifican brindar más lugar a esta metodología en la formación inicial de los docentes.

Finalmente señalamos que, si bien el ABP viene siendo estudiado desde hace muchos años, la construcción de conocimiento sobre la formación docente en dicha metodología resulta aún bastante incipiente. Nuestra propuesta de capacitación, novedosa en el territorio, nos brindó un punto de partida en esta línea de este trabajo que merece consolidarse.

\section{Referencias}

Ayerbe López, J. y Perales Palacios, F. J. (2020). «Reinventa tu ciudad»: aprendizaje basado en proyectos para la mejora de la conciencia ambiental en estudiantes de Secundaria. Enseñanza de las Ciencias, 38(2), 181203.204. https://doi.org/10.5565/rev/ensciencias.2812

Bell, S. (2010). Project-Based Learning for the 21st Century: Skills for the Future. The Clearing House, 83(2), 39-43.

Cascales, A., Carrillo, M. E. y Redondo, A. M. (2017). ABP y Tecnología en Educación Infantil. Píxel-Bit. Revista de Medios y Educación, 50, 201-210.

Coll, C., Mauri, T. y Onrubia, J. (2006). Análisis y resolución de casos problema mediante el aprendizaje colaborativo. Revista de Universidad y Sociedad de Conocimiento, 2(2), 29-41.

Cyrulies, E., Sartarelli, A., Echarri, R. y Vera, S. (2012). Construcción de termotanques solares de bajo costo: un proyecto de voluntariado universitario. Avances en Energías Renovables y Medio Ambiente, 6, 08.13108.141 .

Davini, M. C. (2008). Métodos de enseñanza: didáctica general para maestros y profesores. Buenos Aires, Argentina: Santillana.

Domènech-Casal, J. (2016). Apuntes topográficos para el viaje hacia el \#ABP. Cuadernos de Pedagogía, 742, 59-62.

Domènech-Casal, J., Lope, S. y Mora, L. (2019). Qué proyectos STEM diseña y qué dificultades expresa el profesorado de secundaria sobre Aprendizaje Basado en Proyectos. Revista Eureka sobre Enseñanza y $\begin{array}{lllll}\text { Divulgación le las } & \text { Ciencias } & 16(2), & 2203 .\end{array}$ https://doi.org/10.25267/Rev_Eureka_ensen_divulg_cienc.2019.v16.i2.2203

Ertmer, P. A. y Simons, K. D. (2005). Scaffolding teachers' efforts to implement problem-based learning. International Journal of Learning, 12(4), 319-328.

Gairín Sallan, J. (2011). Formación de profesores basada en competencias. Bordón. Revista de pedagogía, 63(1), 93108.

Gutiérrez-Pérez, J. y Pirrami, F. (2011). Water as Focus of Problem-Based Learning: An Integrated Curricular Program for Environmental Education in Secondary School. US-China Education Review, A 2, $270-280$.

Johari, A. y Bradshaw, A. C. (2008). Project-based learning in an intership program: A qualitative study of related roles and their motivational attributes. Educational Technology Research and Development, 56, 329-359.

Johnson, D. W. y Johnson, R. T. (1995). Teaching students to be peacemakers: Results of five years of research. Peace and Conflict: Journal of Peace Psychology, 1(4), 417-438. 
Larmer, J. y Mergendoller, J. R. (2010). Seven essentials for Project-Based Learning. Educational Leadership, 68(1), 34-37.

Larmer, J., Mergendoller, J. y Boss, S. (2015). Setting the Standard for Project Based Learning: A Proven Approach to Rigorous Classroom Instruction. Alexandria, VA: ASCD.

Perkins, D. (2010). El aprendizaje pleno. Principios de la enseñanza que transforman la educación. Buenos Aires, Argentina: Paidós.

Perrenoud, P. (2004). Diez nuevas competencias para enseñar ( $1^{\circ}$ edic. en español). Madrid, España: Graó.

Roditi, E. (2004). La Résolution de Problèmes Professionnels, Une Modalitè de Formation Continue des Enseignants. París, Francia: Université Paris VII.

Rodríguez-Arteche, I. y Martínez-Aznar, M. M. (2016). Introducing inquiry-based methodologies during initial secondary education teacher training using an open-ended problem about chemical change. Journal of Chemical Education, 93(9), 1528-1535.

Sánchez, J. (2013). Qué dicen los estudios sobre el aprendizaje basado en proyectos. Actualidad Pedagógica.

Recuperado de https://www.estuaria.es/wpcontent/uploads/2016/04/estudios_aprendizaje_basado_en_proyectos1.pdf

Sanmartí, N. (2016). Trabajo por proyectos: ¿Filosofía o metodología? Cuadernos de Pedagogía 472, 44-46.

Tejada Fernández, J. (2009). Competencias docentes. Profesorado. Revista de Currrículum y formación del profesorado, 13(2), 222-234.

Vilches, A. y Gil, D. (2011). El trabajo cooperativo en las clases de ciencias: Una estrategia imprescindible pero aún infrautilizada. Alambique. Didáctica de las Ciencias Experimentales, 69, 73-79.

\section{Agradecimientos}

Al profesor Julio Luna, director de la Escuela Secundaria n. 5 Belgrano Educador, por brindarnos las excelentes instalaciones de dicho establecimiento. Al profesor Javier Servín, exalumno de uno de nosotros, por ser quien contempló el uso del muro Trombe para la enseñanza de la Física en su época de estudiante universitario. A los docentes participantes por demostrar genuino interés en la capacitación en ABP.

\section{Contribución autoral}

a) Concepción y diseño del trabajo; b) Adquisición de datos; c) Análisis e interpretación de datos; d) Redacción del manuscrito; e) revisión crítica del manuscrito.

E. C. y M. S. han contribuido en a, b, c, d, e.

\section{Editora científica responsable}

Mag. Florencia de León 